\title{
A New Technique For Texture Classification Using Markov Random Fields
}

\author{
Mauricio Gomez, Renato A. Salinas
}

\begin{abstract}
This paper proposes, applies and evaluates a new technique for texture classification in digital images. The work describes, as far as possible in a quantitative way, the concept of texture in digital images. Furthermore, we developed an innovative model that allows classifying and characterizing texture in digital images, to be used as a useful tool in noninvasive inspection of visual surfaces. The proposed methodology extracts the statistical order from an image of texture. The extraction of the high statistical order has been made using as a tool Markov Random Fields. The Backpropagation neural net is used for designing a classification module that will serve to test the performance of the configuration histograms, which are based on the statistical order. Furthermore, the research suggests the evaluation of the proposed technique from a qualitative perspective.
\end{abstract}

Keywords: Texture, backpropagation, configuration histograms, classification, Markov Random Fields

\section{Introduction}

Digital image processing is a current subject of research that has being developed for a long time. Of all the invested efforts, multitude of applications have aroused in quite diverse fields such as industrial, medical, spatial, etc. Where very different tasks are made, such as: codification, edge detection, color processing, etc. We are therefore, in front of a subject in constant growth and expansion, that it is giving rise to a high number of projects and publications.

One of the characteristics that are almost always present in an image is the texture, which generally can be associated to different elements from the scene.

In order to be able to apply the concept of texture to the digital analysis of images a quantitative characterization of the texture is required. Nevertheless, it does not exist a precise definition nor a mathematical expression for the quantitative description of this property.

For the analysis of the texture, there exist three widely used formulations in image processing, they are: statistical [1], structural and spectral [2]. The statistical formulation indicates if an image is smooth, rustic, granulated, etc. [1]. The structural technique, on the other hand, indicates the primitive features that exist in the image, such as regularity of parallel lines; and the spectral technique is based on the energy properties and it is used mainly to detect global regularity in an image, indicating small peaks of high energy in its spectrum [2]. These techniques can used separately or jointly to detect different textures.

The detection of textures has wide spectra of applications, mainly in the following areas:

- Medicine: in several types of images (x-rays, ultrasound, magnetic resonance, etc.); the properties of the texture are important for the diagnosis. Cancer frequently is characterized by analysis of texture in different types of medical images; also techniques of texture have been applied satisfactorily for the detection of abnormalities in mammographic images [3].

- Remote sensors: numerous formulations for recognition of textures in remote sensors have been proposed. Their applications include: land classification, cloud classification, recognitions of seismic patterns, etc. 
- Industrial inspection: in industrial processes, the detection of texture in defective manufactured products or natural materials is of crucial importance. The manual inspection frequently is tedious and laborious, for that reason, the automation is very useful [4].

However, none of these classic descriptions leads to a quantitative measurement universally accepted of texture. Due to this difficulty, research in this field continues being interesting until a method be obtained that is satisfactory for most of the applications.

This research is an attempt to describe in a quantitative way, the concept of texture in digitized images. Furthermore, it develops an innovative model that allows to classify and to characterize textures in digitized images, and it can be used as a useful tool in noninvasive visual inspection.

\section{Models of the texture}

In the image processing literature, texture is often defined as the spatial interaction between the values of pixels. That is to say, the texture analysis attempts capturing its visual characteristics in an analytical way, in order to be able to model mathematically those spatial interactions. Modeling successfully the visual characteristics, we will be able to associate and to discriminate different textures analytically. This type of analysis will require of a number and type of textures previously known. This model only needs to capture sufficient textural characteristics on a training set to classify the texture, however, the procedure is best adapted for textures that belong to the training set.

Of these models of understanding of texture, there are four of them that stand out in the literature of digital image processing.

Statistical models of texture: It is based on a set of features to represent the characteristics of the texture of an image. Those features are contrast, correlation, entropy, etc. They are usually derived from "measurement of the gray level of the image", "it differentiates from the values of gray" or "cooccurrence matrix" [1]. The characteristics are selected in heuristic form; nevertheless, an image similar to the analyzed one cannot be recreated using some measurement of the set of features.

Structural models of the texture: Some textures can be seen as two-dimensional patterns, composed of a set of primitives or sub-patterns, which are organized according to a certain rule of positioning. Examples of such textures are: brick walls and mosaics; the primitives used are areas of constant gray level, lines, curves and polygons. The correct identification of those primitives is quite difficult. However, if the textures primitives are identified completely, then it is possible to recreate the texture from the primitives. A work using a structural model is indicated in [4].

Models of signal processing for the texture: psychological researchers have given evidence that the human brain analyzes the frequency of the images. The texture is specially fit for this type of analysis due to its properties. Most of the techniques try to process certain characteristics from filtered images, which are used in tasks of classification and segmentation. Among the methods we can mention: filters in the spatial domain, Fourier analysis, wavelets and Gabor filters. Works using this type of formulation are given in [5].

Stochastics models of the texture: a texture is assumed to be the realization of a stochastic process, which is governed by some parameters. The analysis is executed, defining a model and considering the parameters. This way, the stochastic processes can be reproduced from the model and associated to the parameters. The estimation of the parameters can serve to classify and to segment textures. This type of model offers a good possibility to recreate realistic examples of natural textures. Stochastic formulation of the texture is used in [6]. 


\section{Our model of texture}

As seen previously, there are several problems associated with modeling texture. A key factor in our model of texture analysis is that it be able to distinguish between different textures. Conventional techniques have been based on prior knowledge of the number and types of textures that are desired to analyze. In our model we shall use the same philosophy of analysis. Therefore, we need to construct a model that can take a texture and capture the totality of the characteristics that distinguish that group of textures. But, in which way we can capture the real characteristics that distinguish a group of textures? And, which will be our approach in the direction of constructing such characteristics? We believe that an understanding of the statistical order applied to images of digitized textures will allow us to capture the real characteristics that distinguish natural textures. We know that the statistical understanding of the texture was proposed by Julesz as early as 1962. The statistical order in the understanding of the texture, applied to digital processing of images has been used in [1], where second order statistics are used to define 14 types of textural features from the cooccurrence matrix. In the texture models that use the auto-models and multilevel logistic models also use second order statistics; nevertheless, they have not provided a base of understanding applicable to natural textures, although in [7] modest results were reached using a Gaussian model of Markov Random Field that use precisely second order statistics. Models that have managed to recreate natural textures using several multiresolution filters do not use third or higher statistical orders, and even more, they become indefinite (ill-defined) if the election of the filters is globally optimal for all the analyzed textures. Julesz in 1962 had already indicated the existence of textural information in the high statistical order.

In such a way, starting from the knowledge already generated and using the approach of the human visual perception proposed by Julesz, is that we have raised our hypothesis applied to textures in digitized images, which establishes that to model textures, models of different statistical orders are required and for it we have chosen the Markov Random Fields as the basis of our model of texture due to its flexibility in defining the statistical dimension and the order.

\section{Markov Random Fields}

The theory of the Markov Random Fields (MRF) provides suitable tools for us to model context dependent entities such as pixels or other characteristics of spatial correlation [8], [9]. Markov Random Fields are defined with respect to a system of neighborhoods. The mathematical interpretation of the model is defined with respect to the corresponding set of cliques. In this research, a systematic method for the total extraction of the set of local cliques has been used from any system of neighborhood in which the Markov Random Field can be defined.

The fundamental property of a MRF is that given a point $j$ on lattice, the probability of that point, given the values of all the other points of the lattice, is the same as the probability of that point $j$, given only the values of the neighboring points to $j[8,9]$. In other words, the MRFs are characterized by a conditional probability function defined with respect to a system of neighborhoods.

We denote a set of sites on a lattice by $S$ and the system of vicinities on $S$ as $G=\left\{G_{s}, s \in S\right\}$, where $G$ is the set of neighbors for $s$ so that $G s G_{s} \subset S, s \notin G_{s}$.

Given the variable $X_{s}$ in site $s$ with value $x_{s}$ the function of conditional probability of a Markov Random Field with its respective system of vicinity $G$ is defined by the theorem of Hammersley and Clifford [9] as:

$$
P\left(X_{s}=x_{s} \mid X_{r}=x_{r}, r \in G_{s}\right)=\frac{1}{Z_{s}} \exp \left\{-\sum_{c} V_{c}(x)\right\}
$$

Where $Z_{s}$ is a constant and $V c$ is a potential function defined on clique $C$. The sum is over all cliques in the local set of cliques $C s$. 


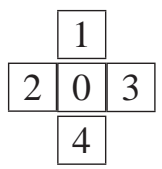

\begin{tabular}{|l|l|l|}
\hline 1 & 2 & 3 \\
\hline 4 & 0 & 5 \\
\hline \hline 6 & 7 & 8 \\
\hline
\end{tabular}

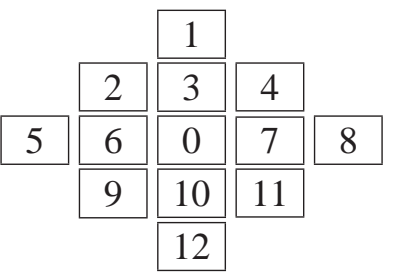

Figure 1: Three different neighborhoods.

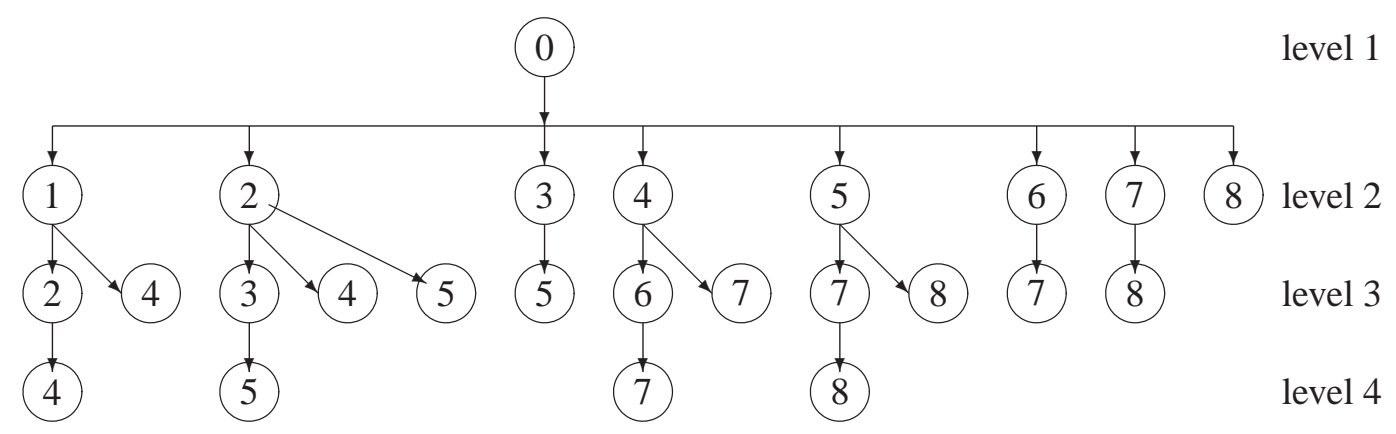

Figure 2: Tree diagram for the $3 \times 3$ neighborhood.

The theorem of Hammersley and Clifford, indicates that for a system of neighborhoods is necessary that $s \in G_{r} \Longleftrightarrow r \in G_{s}$. Three different neighborhoods are shown in figure 1 .

Given a system of neighborhoods $G$, a clique is a set $C \subseteq S$ so that $s, r \in C, s \neq r$, then $s \in G_{r}$. That is, all the pairs of different sites in a clique are neighbors. In such a way that the local set of cliques for site $s$ is defined as:

$$
C_{s}=\{C \subseteq S: s \in C\}
$$

The method used for the extraction of the local set of cliques from a system of neighborhoods of a MRF, is based on a structure of a tree diagram [10]. The root of the tree represents only a site, whereas the branches in the first level represent all the pairs of connections between the site and each one of the components of the neighborhoods. The other branches of the highest levels represent connections of high order that form more complex cliques. Each level in the tree diagram will be represented by a node, which is indicated by a number within the neighborhood previously defined. For example, the tree diagram for the neighborhood of the figure 1.b is shown in figure 2.

\section{Characterization of texture using order statistics}

In order to characterize the texture of a digitized image, we will use a vector called of directional homogeneity [10], which considers the relation between the pixel corresponding to a site $s$ and all the remaining pixels of neighborhood $G_{s}$. The relation to be established between the site and the other pixels of the neighborhood will depend on the type of clique and the statistical order used. Figure 1 shows different types from neighborhood with site $s$.

Let us suppose that $X$ is a random field of the class described in [8,9], with a space of discrete range $Z=\left\{z_{k}, k=1, \ldots, M\right\}$. Let us also suppose that we have an instance $x$ of this one random field that 


\begin{tabular}{|c|c|c|}
\hline V1 & U2 & V2 \\
\hline U1 & S & U3 \\
\hline V4 & U4 & V3 \\
\hline
\end{tabular}

Figure 3: Site s and its neighborhood.

can be used for the estimation of parameters of the distribution. Figure 3 shows a site $(i, j)$ and its system of neighborhood with the notation employed.

Furthermore, we define as homogeneity function the function I such that:

$$
I\left(z_{1}, \ldots, z_{k}\right)=\left\{-1, \text { if } z_{1}=z_{2}=\ldots=z_{k} ; 1,\right. \text { otherwise }
$$

This homogeneity function, for the case of cliques of first order will be defined as:

$$
I(s)=\left\{-1 \text { if } s=z_{m} ; 0,\right. \text { otherwise }
$$

Using the terms defined until now, we can express the potential functions of a Gibbs distribution,

$$
V(s, E, \theta)=\phi(s, E)^{T} \theta
$$

Where is the vector of parameters and the vector that we will call of directional homogeneity. For example, for the neighborhood of figure 3 the component of the vector, using the tree of cliques (see figure 2) is defined as:

$$
\phi_{3}=\left\{\begin{array}{c}
\left(I\left(s, u_{2}, v_{2}\right)+I\left(s, u_{4}, u_{3}\right)+I\left(s, u_{1}, v_{4}\right)\right), \\
\left(I\left(s, u_{4}, v_{3}\right)+I\left(s, u_{2}, u_{3}\right)+I\left(s, u_{1}, v_{1}\right)\right), \\
\left(I\left(s, u_{2}, v_{1}\right)+I\left(s, u_{1}, u_{4}\right)+I\left(s, u_{3}, v_{3}\right)\right), \\
\left(I\left(s, u_{1}, u_{2}\right)+I\left(s, u_{4}, v_{4}\right)+I\left(s, u_{3}, v_{2}\right)\right)
\end{array}\right\}
$$

The set of components $\phi_{k}$ of vector $\phi$ represents the different statistical order extracted from the image, where $k$ is the statistical order with $k \in\{1,2,3,4\}$ for a neighborhood of second order like the one shown in figure 3.

\section{Direct measurement of the statistical order}

This section presents our proposal to characterize digitized images of texture that makes use of the properties of vector $\phi$ in each point of the digitized image.

Taking the expressions 3, 4and 5 for a second order neighborhood (figure 3) and applying them to the calculation of the components of vector $\phi$, we see that the values that can take components are the following:

$$
\begin{gathered}
\phi_{1} \in\{0,1\} \\
\phi_{2} \in\{-2,0,2\} \\
\phi_{3} \in\{-3,-1,1,3\} \\
\phi_{4} \in\{-1,1\}
\end{gathered}
$$

Of the possible values that can take different components $\phi_{k}$, we establish equivalence between the value and its respective binary, ternary or tetranary value, as corresponds. Table 1 illustrates some examples of equivalences. 
Table 1: Equivalence between the value and ternary number.

\begin{tabular}{|c|c|}
\hline Value & Ternary Number \\
\hline-2 & 0 \\
\hline 0 & 1 \\
\hline 2 & 2 \\
\hline
\end{tabular}
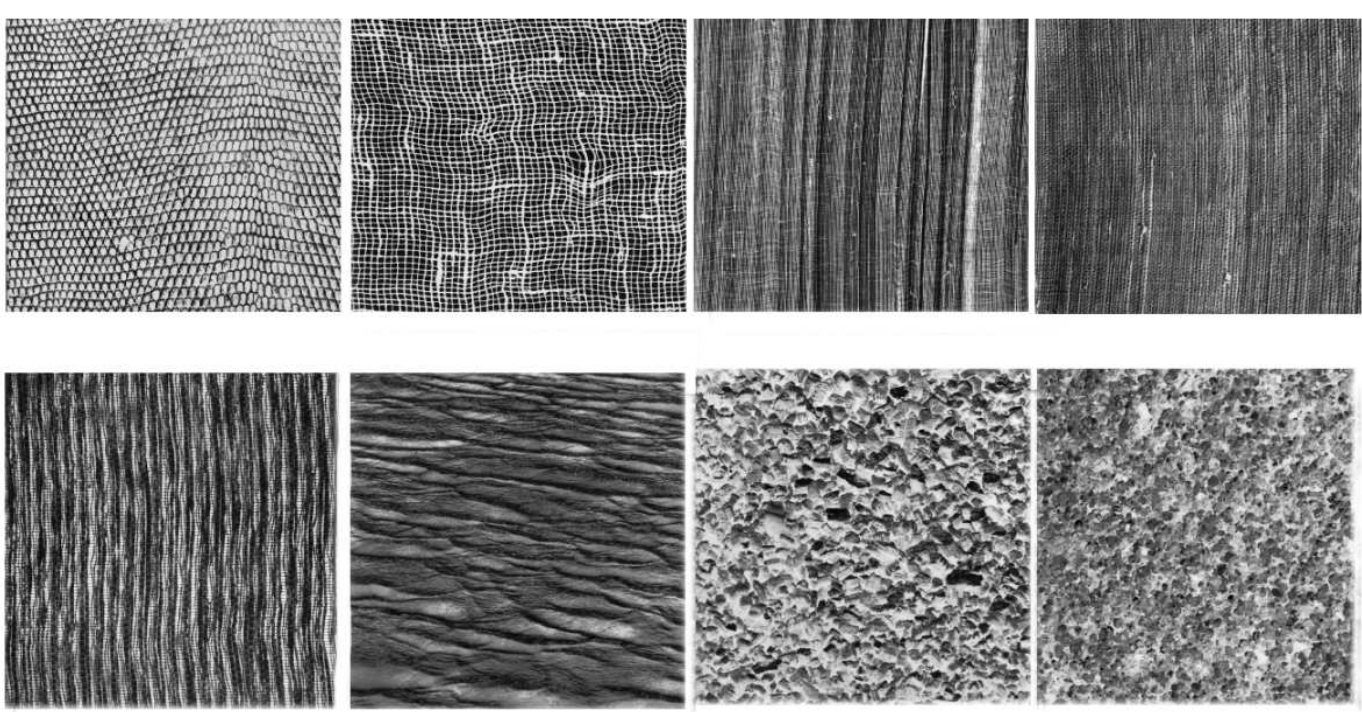

Figure 4: Brodatz textures used in our experiments. Top row: D22, D103, D105 and D79; Bottom row: D76, D37, D5 and D28.

Now, our interest is only in the values that take the components of vector $\phi$, that is why we will work with a decimal number, instead of considering its vector array.

This way, given a digitized image of texture and applying the different statistical orders through the different cliques used, we will obtain histograms of frequency versus directionality, which will be different and particular for each of the analyzed textures.

\section{Classification of textures using histograms of configurations}

The process of texture classification will be based on the histograms of configurations proposed in the previous section. In the present work we have used a set of 8 classes of textures that have been obtained from the album of textures of Brodatz [11]. Each image has a dimension of 640x640 with 256 gray levels ( 8 bits/pixel). The images of textures used are shown in Figure 4.

For each of these images, 32 sub-images of $64 \times 64$ pixels have been extracted. For each sub-image, the histogram of configurations has been computed. In figures 6,7 and 8 , can be seen some of those histograms.

The form in which the 32 sub-images have been extracted is explained in a scheme (figure 5) that represents the 32 sub-images taken from one of the images shown in figure 4 . The white squares represent the 32 sub-images and the dark background represents the greater image of the texture (figure 5). Images with an asterisk have been taken to construct a characteristic vector, which will be used to train the 


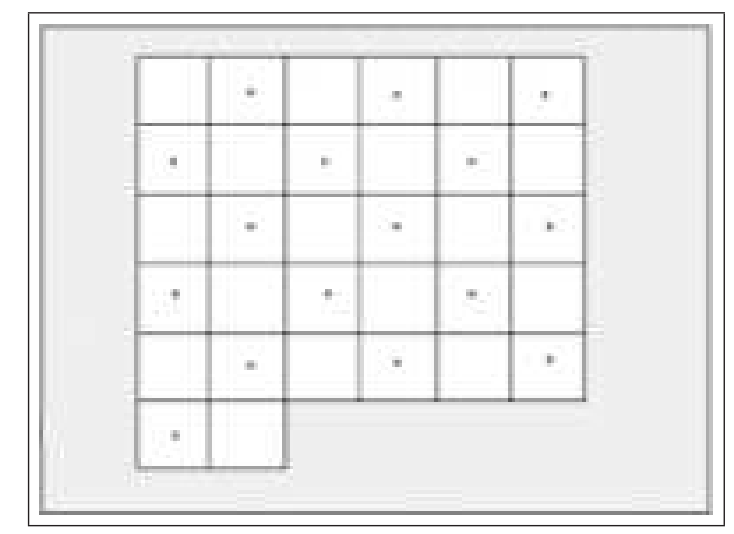

Figure 5: Layout employed to select the training and validation subimages. Each cell represents a subimage, and every cell containing an asterisk is used for training.

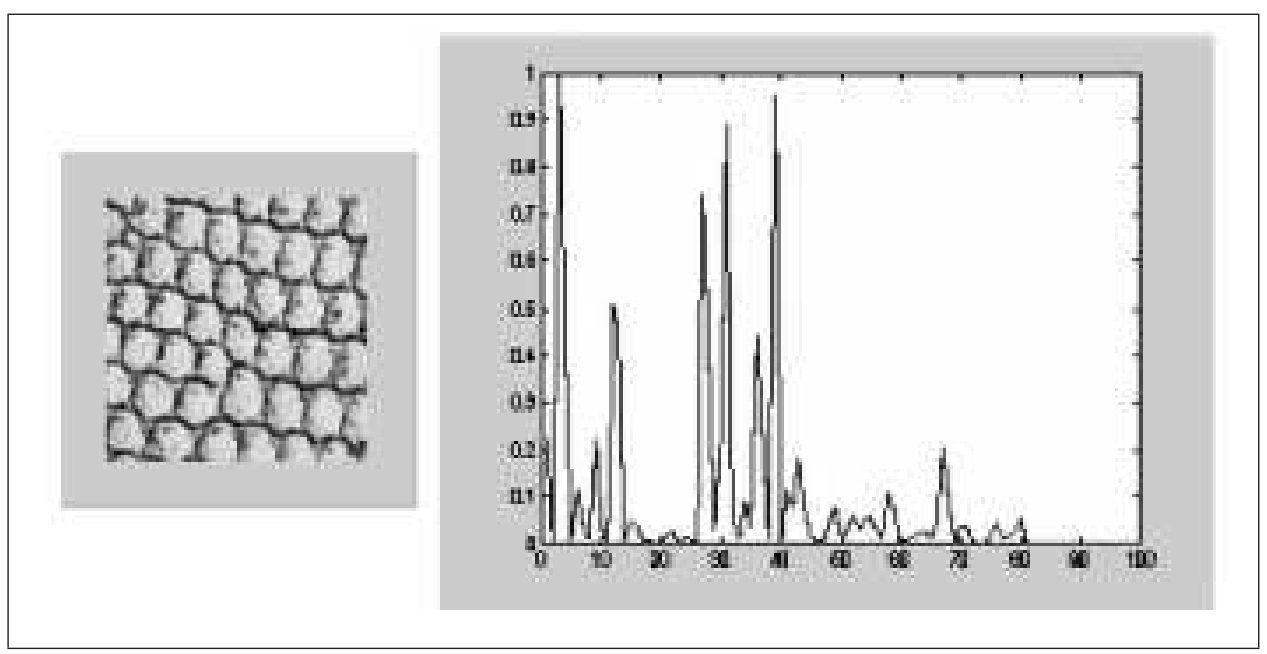

Figure 6: Texture D22 and its corresponding configuration histogram.

classification module. This procedure has been performed with the eight images of textures shown in figure 4 . The sub-images without asterisk in figure 5 are used to test the performance of the texture classification module.

The architecture that has been developed for classification of the texture consists of four basic blocks (see figure 9): a) Extraction of characteristic vector. The purpose of this block is to construct a vector made of the frequencies of the histogram of configurations used; b) Classification: the objective of this block is to classify the different characteristic vectors, that is to say, the information contained in the digitized input image (texture). For this block it has been proposed using a backpropagation (BP) neural network; c) Decision: this block makes the final decision from data generated by the classification block; d) Data Base: it stores parameters and models to control adaptively the operation of the various blocks.

Once determined the configuration histograms for different statistical orders for the 32 sub images and for each texture category, each configuration histogram is evaluated with respect to performance. 


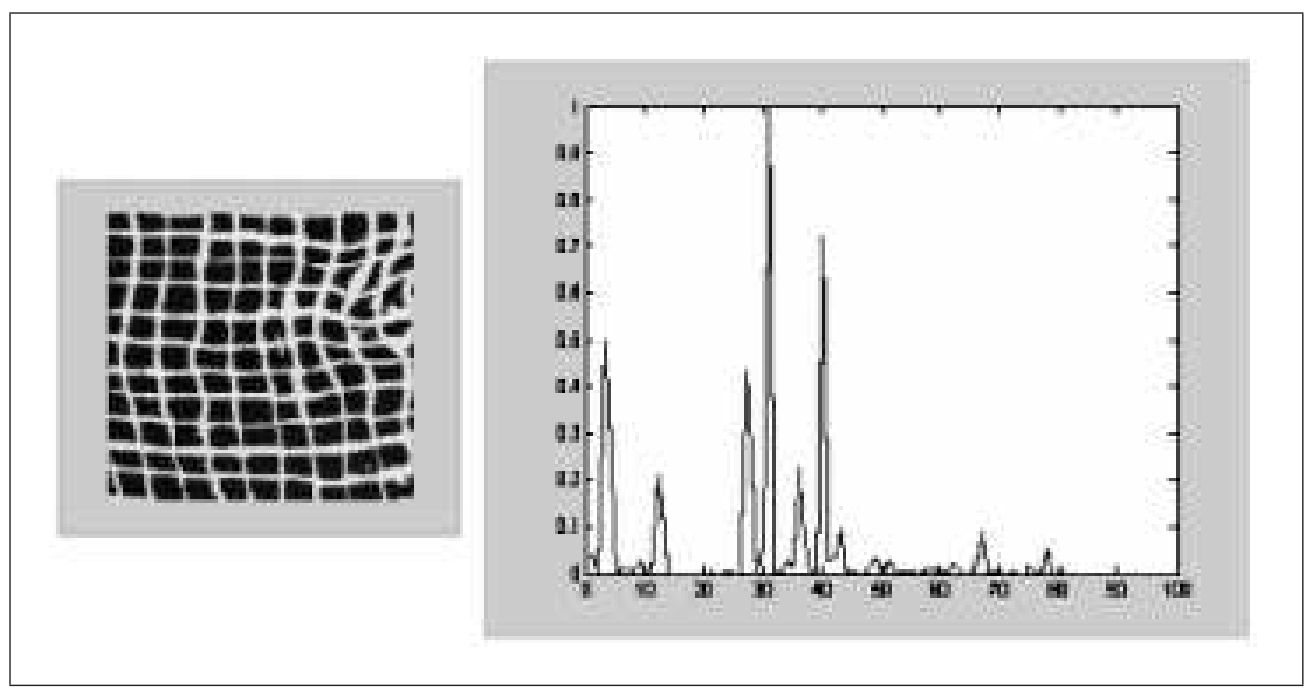

Figure 7: Texture D103 and its corresponding configuration histogram.

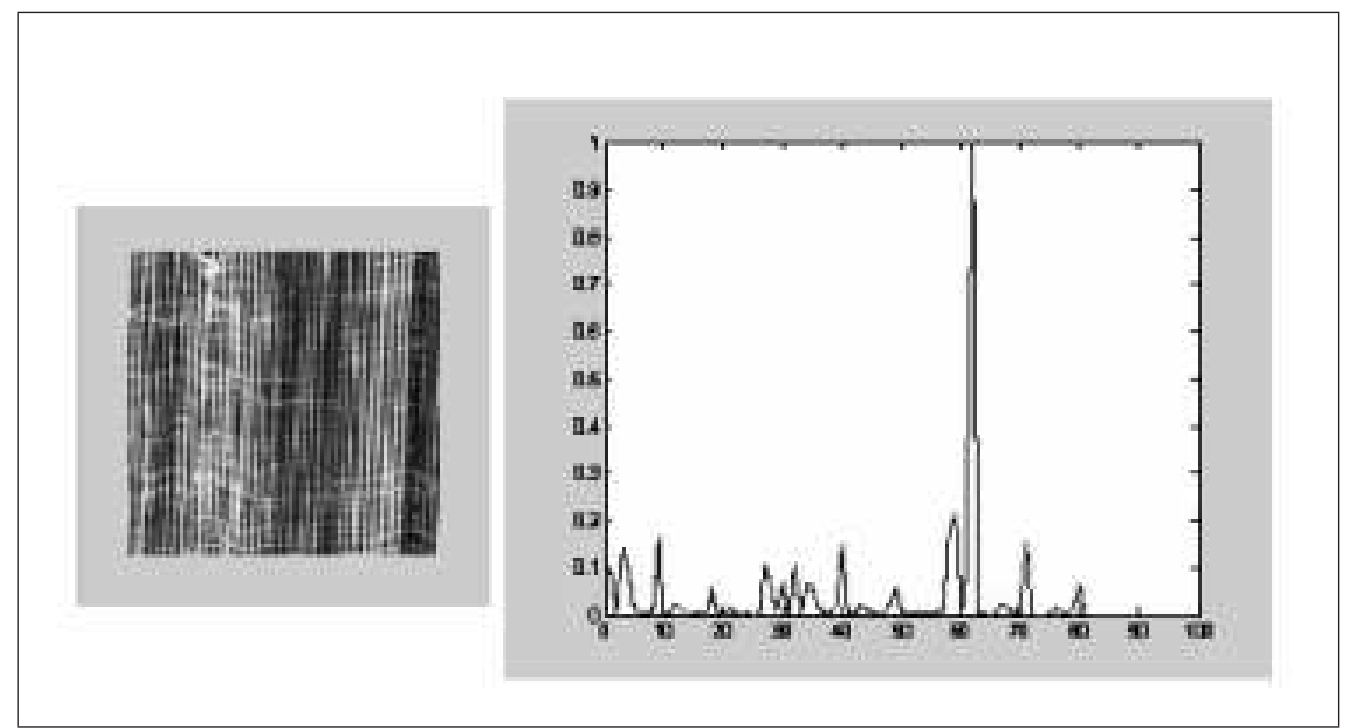

Figure 8: Texture D105 and its corresponding configuration histogram. 


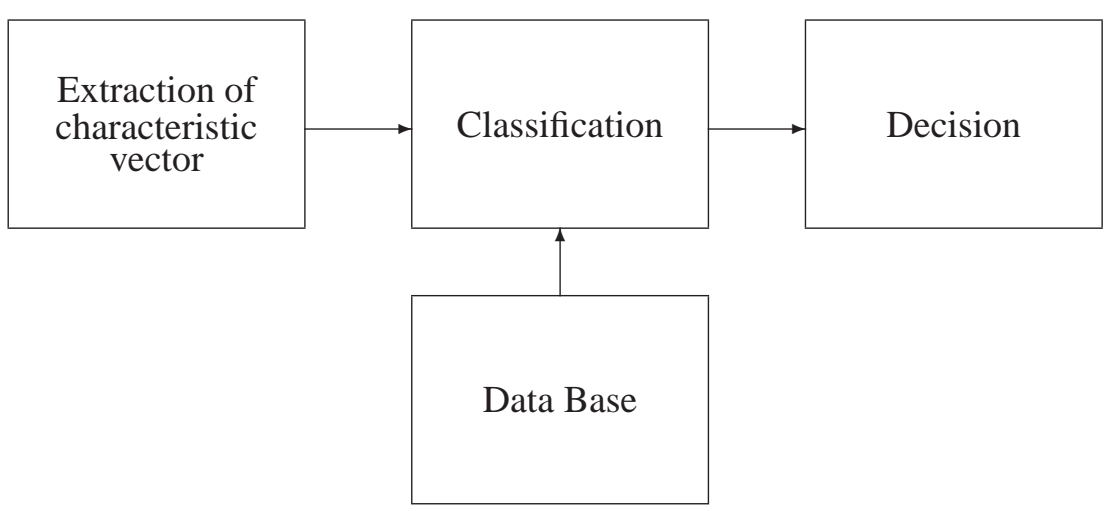

Figure 9: Block diagram of the proposed system.

Table 2: Proposed binary codes for the seven output nodes.

\begin{tabular}{|c|c|c|c|c|c|c|c|}
\hline Texture & 1st & 2nd & 3rd & 4th & 5th & 6th & 7th \\
\hline D22 & 0 & 0 & 0 & 0 & 0 & 0 & 0 \\
\hline D5 & 0 & 0 & 0 & 0 & 0 & 0 & 1 \\
\hline D79 & 0 & 0 & 0 & 0 & 0 & 1 & 0 \\
\hline D76 & 0 & 0 & 0 & 0 & 1 & 0 & 0 \\
\hline D28 & 0 & 0 & 0 & 1 & 0 & 0 & 0 \\
\hline D37 & 0 & 0 & 1 & 0 & 0 & 0 & 0 \\
\hline D105 & 0 & 1 & 0 & 0 & 0 & 0 & 0 \\
\hline D103 & 1 & 0 & 0 & 0 & 0 & 0 & 0 \\
\hline
\end{tabular}

The classification module, is built using a BP neural net that is trained with 16 sub images and validated with the remaining 16 unused sub images.

A BP neural net is trained with a predefined set of input-output pairs. Each input pattern (vector) is applied to the input layer, and it propagates through the successive layers until it generates a given output. This current output is compared against the target output to obtain the so called error vector. These errors are back propagated, beginning at the output layer and then propagated to the hidden layers until the input layer is reached. Based upon an error minimization procedure, the connection weights of each neuron are modified, in such a way as to reduce the final error the next time the same input pattern be presented to the network.

For this research, a BP neural net with 3 layers has been used. The number of nodes of the input layer is the same as the number of components of the characteristic vector. For the hidden layer, from 12 to 16 nodes have been used, and finally, for the output layer, 7 neurons with proper codification have been employed. Table 2 shows the proposed code for the seven output nodes.

The BP neural net has been programmed and trained using Matlab( rithms have been evaluated for weight modification: gradient descent (traingd) and Levenberg-Marquardt (trainlm). 
Table 3: Results using a 3x3 neighborhood and second order histograms.

\begin{tabular}{|c|c|c|c|c|c|c|c|c|c|}
\hline Texture & D22 & D103 & D105 & D79 & D76 & D37 & D5 & D28 & Total \\
\hline D22 & $\mathbf{1 6}$ & & & & & & & & \\
\hline D103 & & $\mathbf{1 4}$ & & & & & & & \\
\hline D105 & & 2 & $\mathbf{1 1}$ & & 2 & & 1 & & \\
\hline D79 & & & 3 & $\mathbf{1 2}$ & & & & 1 & \\
\hline D76 & & & 2 & & $\mathbf{1 3}$ & & 2 & 2 & \\
\hline D37 & & & & 3 & & $\mathbf{1 6}$ & & & \\
\hline D5 & & & & & 1 & & $\mathbf{1 1}$ & 3 & \\
\hline D28 & & & & 1 & & & 2 & $\mathbf{1 0}$ & \\
\hline \% & 100 & 87.5 & 68.8 & 75 & 81.3 & 100 & 68.8 & 62.5 & $\mathbf{8 0 . 4 7}$ \\
\hline
\end{tabular}

Table 4: Results using a $5 \times 5$ neighborhood and third order histograms.

\begin{tabular}{|c|c|c|c|c|c|c|c|c|c|}
\hline Texture & D22 & D103 & D105 & D79 & D76 & D37 & D5 & D28 & Total \\
\hline D22 & $\mathbf{1 6}$ & & & 1 & & & & & \\
\hline D103 & & $\mathbf{1 4}$ & & & & & & & \\
\hline D105 & & 1 & $\mathbf{1 3}$ & & 1 & & & & \\
\hline D79 & & & & $\mathbf{1 2}$ & & & & & \\
\hline D76 & & & 2 & & $\mathbf{1 3}$ & & & 1 & \\
\hline D37 & & & & 3 & & $\mathbf{1 6}$ & & & \\
\hline D5 & & 1 & & & 2 & & $\mathbf{1 3}$ & 1 & \\
\hline D28 & & & 1 & & & & 3 & $\mathbf{1 4}$ & \\
\hline \% & 100 & 87.5 & 81.3 & 75 & 81.3 & 100 & 81.3 & 87.5 & $\mathbf{8 6 . 7 2}$ \\
\hline
\end{tabular}

\section{Experimental results using histograms of configurations}

Table 3 shows the classification results obtained using a $3 \times 3$ neighborhood and histograms of configurations of second order statistics. In this case the classification module was built with 16 hidden layer neurons, and trained for 500 epochs.

Later on, the performance was evaluated with $5 \times 5$ neighborhoods and third order histograms. Table 4 shows the results of this experiment.

From the above results, we can confirm that the histograms of configurations are sufficiently representative of the textures analyzed using different statistical orders. Furthermore, we achieve a classification rate of $86.72 \%$ while employing third statistical order.

\section{Summary and Conclusions}

A new texture classification method has been developed, based on the extraction of the statistical order from a digitized texture image. This later operation has been performed using the set of local cliques from a neighborhood.

The use of histograms of configurations in the process of texture classification has been quite successful, achieving a classification rate of around $87 \%$.

The decision of employing a BP neural network was also adequate for the classification. Best results 
were reached when training the neural net with the Levenberg-Marquardt method.

The proposed classification architecture requires of just a few features, thus achieving a reduction in training time and smaller computational efforts during the classification stage.

The work developed so far constitutes the initial stage of a series of applications and works related to texture and non-destructive visual inspection of materials.

\section{References}

[1] Haralick R., Shanmugam K. and Dinstein I., Textural Features for Image Classification, IEEE Trans. on Systems, Man, and Cybernetics, Vol. SMC-3, No . 6, pp. 610-621, 1973.

[2] Hsin H., Texture Segmentation Using Modulated Wavelet Transform, IEEE Trans. on Image Processing, Vol 8. $\mathrm{N}^{o} .7,2000$.

[3] Hernández L., Torrealba V. and Reigosa A., Clasificación Automática del carcinoma de la mama, mediante un sistema de reconocimiento basado en redes neuronales, Memorias II Congreso Latinoamericano de Ingeniería Biomédica, La Habana, Cuba, 2001.

[4] Mery D., Da Silva R., Calôva L. and Rebello J., Detección de fallas en piezas fundidas usando metodología de reconocimiento de patrones, 3rd Panamerican Conference for Nondestructive Testing, Rio de Janeiro, Brazil, 2003.

[5] Bader D., JáJá J. and Chellapa R., Scalable Data Parallel Algorithms for Texture Synthesis using Gibbs Ramdon Fields, IEEE Transactions on Image Processing, vol. $4 \mathrm{~N}^{o} .10$, pp. 1456-1460, 1995.

[6] Pun C. and Lee M., Rotation Invariant Texture Classification Using a Two Stage Wavelet Packet Features Approach, IEE Proc. Vis. Image Signal Process, vol. No 148, No6, pp 422-428, 2001.

[7] Manjunath B., Simchony T. and Chellappa R., Stochastic and Deterministic Networks for Texture Segmentation, IEEE Transactions on Acoustics Speech and Signal Processing, vol. N ${ }^{o} 6$, pp. 10391047, 1990.

[8] Li S., Modeling Image Analysis Problems Using Markov Random Fields, Handbook of Statistics, vol. $\mathrm{N}^{o} 20,2000$.

[9] Li S., Markov Random Fields Models in Computer Vision, Proceedings of IEEE Computer Society Conference on Computer Vision and Pattern Recognition, pp 866-869, 1994.

[10] Gómez M., Modelación y clasificación de texturas utilizando Campos Aleatorios de Markov, Master Thesis, Electrical Engineering, Universidad de Santiago de Chile, Santiago, Chile, 2004.

[11] Brodatz P., Textures. New York: Dover, 1966.

Renato Salinas, Mauricio Gomez

Universidad de Santiago de Chile

Electrical Engineering Department

Ave. Ecuador 3519, Santiago, Chile

E-mail: rsalinas@lauca.usach.cl, mauriciogomez@esfera.cl 